\title{
Left atrial remodeling and mechanical dysfunction in hypertrophic cardiomyopathy
}

\author{
Kyung-Jin Kim', Hong-Mi Choi ${ }^{1 *}$, Yeonyee E Yoon ${ }^{1}$, Jun-Bean Park ${ }^{3}$ Jin Joo Park', Hyo Eun Park², Seung-Pyo Lee ${ }^{3}$, \\ Goo-Young Cho ${ }^{1}$
}

From 19th Annual SCMR Scientific Sessions

Los Angeles, CA, USA. 27-30 January 2016

\section{Background}

Patients with hypertrophic cardiomyopathy (HCM) and LA enlargement have more serious cardiovascular events. This study sought to determine the contribution of left atrial (LA) remodeling and mechanical function to hypertrophic cardiomyopathy (HCM) by comparing HCM patients to age- and gender-matched control subjects and young healthy control subjects, and also to assess whether LA remodeling and mechanical function including global strain are related to the characteristics of HCM.

\section{Methods}

A total of 79 patients with HCM who underwent both 2Dspeckle tracking echocardiography and cardiac MRI were included ( $54 \pm 12$ years; 60 men) and compared to 79 ageand gender-matched control subjects and 20 young healthy control subjects. LA diameter and volume, expansion index for reservoir function, active emptying fraction for pump function, global longitudinal LA strain were measured. The type of HCM, the presence and extent of late gadolinium enhancement (LGE) in left ventricular

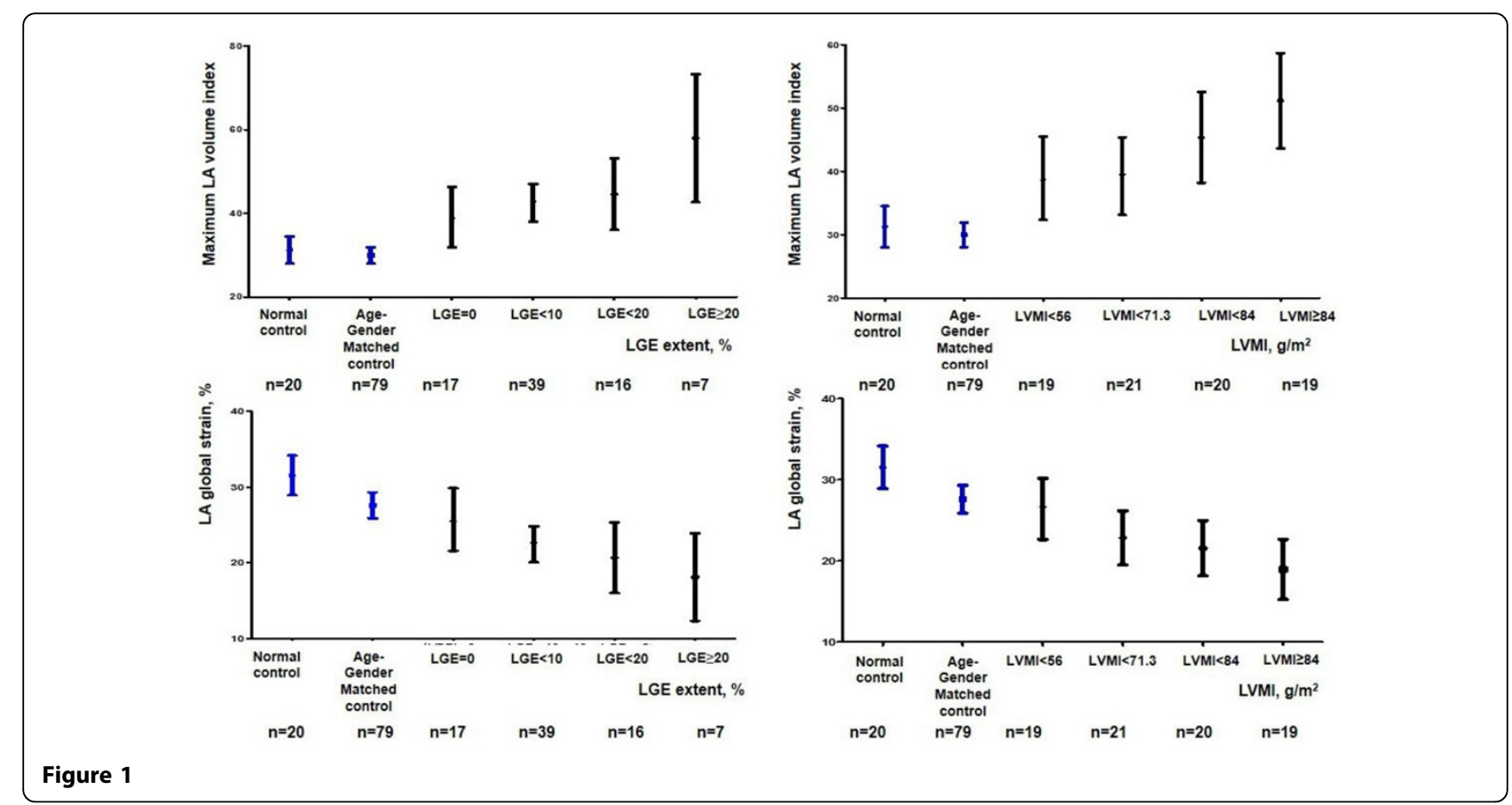

'Division of Cardiology, Cardiovascular Center, Seoul National University

Bundang Hospital, Seongnam city, Korea (the Republic of)

Full list of author information is available at the end of the article

C 2016 Kim et al. This is an Open Access article distributed under the terms of the Creative Commons Attribution License (http:// creativecommons.org/licenses/by/4.0), which permits unrestricted use, distribution, and reproduction in any medium, provided the original work is properly cited. The Creative Commons Public Domain Dedication waiver (http://creativecommons.org/publicdomain/ zero/1.0// applies to the data made available in this article, unless otherwise stated. 
(LV) myocardium and LV mass index by cardiac magnetic resonance imaging was evaluated.

\section{Results}

When compared to age- and gender-matched control subjects and young healthy control subjects, HCM patients showed an increased LA volume index $\left(30.0 \pm 8.7 \mathrm{ml} / \mathrm{m}^{2}\right.$ vs $31.3 \pm 7.0 \mathrm{ml} / \mathrm{m}^{2}$ vs $43.6 \pm 15.1 \mathrm{ml} / \mathrm{m}^{2}, P$ value < $0.001)$, impaired reservoir function $(111 \pm 42 \%$ vs $150 \pm$ $57 \%$ vs $94 \pm 41 \%, P$ value $<0.001)$, decreased LA global strain $(27.6 \pm 7.7 \%$ vs $31.5 \pm 5.6 \%$ vs $22.4 \pm 7.9 \%$, $P$ value $<0.001)$. LA volume index and global strain demonstrated graded association with the extent of LV myocardial scar and LV mass index evaluated by cardiac MRI. (Figure) However, LV mass index was the only independent determinant of LA enlargement and LA global strain rather than LGE extent (LA maximum volume index: $\beta=0.358, P$ value $=0.003$, LA global strain: $ß=-0.295, P$ value $=0.021)$.

\section{Conclusions}

In $\mathrm{HCM}$, the strongest determinant of LA remodeling and dysfunction is left $\mathrm{LV}$ mass index rather than the extent of LV myocardial scar.

\section{Authors' details}

${ }^{1}$ Division of Cardiology, Cardiovascular Center, Seoul National University Bundang Hospital, Seongnam city, Korea (the Republic of). ${ }^{2}$ Healthcare System Gangnam Center, Seoul National University Hospital, Seoul, Korea (the Republic of). ${ }^{3}$ Division of Cardiology Department of Internal Medicine, Seoul National University Hospital, Seoul, Korea (the Republic of).

Published: 27 January 2016

doi:10.1186/1532-429X-18-S1-P288

Cite this article as: Kim et al:: Left atrial remodeling and mechanical dysfunction in hypertrophic cardiomyopathy. Journal of Cardiovascular Magnetic Resonance 2016 18(Suppl 1):P288. 